\title{
Erratum to: Prognostic significance of late gadolinium enhancement quantification in cardiac magnetic resonance imaging of hypertrophic cardiomyopathy with systolic dysfunction
}

\author{
Akira Funada $^{1} \cdot$ Hideaki Kanzaki $^{1} \cdot$ Teruo Noguchi $^{1} \cdot$ Yoshiaki Morita $^{2} \cdot$ \\ Yasuo Sugano $^{1}$ Takahiro Ohara $^{1}$ - Takuya Hasegawa ${ }^{1}$ - Hiromi Hashimura ${ }^{2,3}$. \\ Hatsue Ishibashi-Ueda ${ }^{3} \cdot$ Masafumi Kitakaze $^{1,4} \cdot$ Satoshi Yasuda $^{1} \cdot$ Hisao Ogawa $^{1}$. \\ Toshihisa Anzai ${ }^{1}$ \\ Published online: 24 June 2015 \\ (C) Springer Japan 2015
}

\section{Erratum to: Heart Vessels}

\section{DOI 10.1007/s00380-015-0670-4}

Unfortunately, the scale of the vertical axis of Fig. $2 b$ and the scale of the horizontal axis of Fig. 5b appeared incorrectly in the article above cited. The corrected Figs. $2 \mathrm{~b}$ and $5 \mathrm{~b}$ are given below.

The online version of the original article can be found under doi:10.1007/s00380-015-0670-4.

Akira Funada

a-funada@e-mail.jp

1 Department of Cardiovascular Medicine, National Cerebral and Cardiovascular Center, 5-7-1 Fujishiro-dai, Suita, Osaka 565-8565, Japan

2 Department of Radiology, National Cerebral and Cardiovascular Center, Suita, Osaka, Japan

3 Department of Pathology, National Cerebral and Cardiovascular Center, Suita, Osaka, Japan

4 Department of Clinical Research and Development, National Cerebral and Cardiovascular Center, Suita, Osaka, Japan 
Fig. 2 Relationship between LVEF and the extent of LGE. a LVEF and \% LGE with a threshold of 2SD, b LVEF and $\%$ LGE with a threshold of 6SD. \% LGE with a threshold of 6SD was inversely related to $\operatorname{LVEF}(r=-0.44 ; p=0.002)$, but that with a threshold of 2SD was not $(r=-0.28, p=0.060)$. Abbreviations as in Table 1 (a)

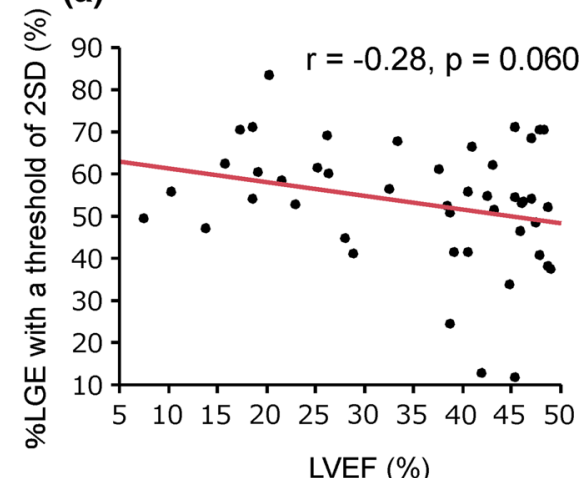

(b)

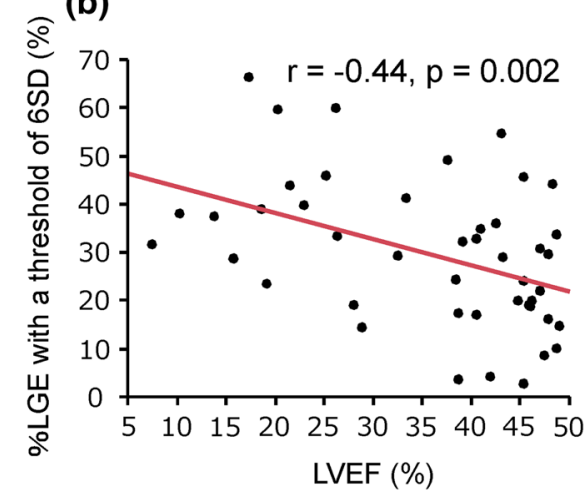

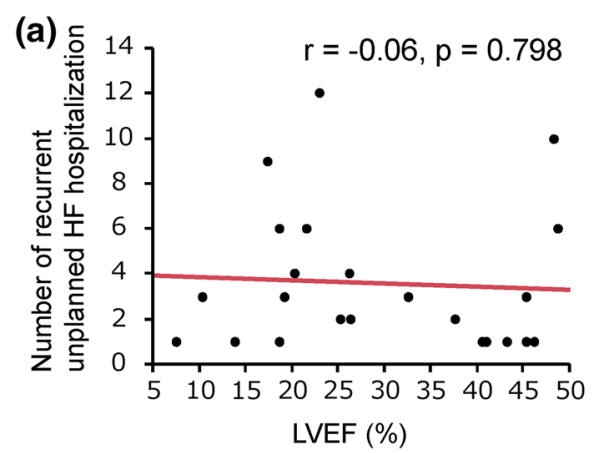

Fig. 5 Relationship between LVEF, the extent of LGE, and number of recurrent unplanned heart failure hospitalizations. a LVEF and number of recurrent unplanned HF hospitalizations, b \% LGE with a threshold of 6SD and number of recurrent unplanned HF hospi-

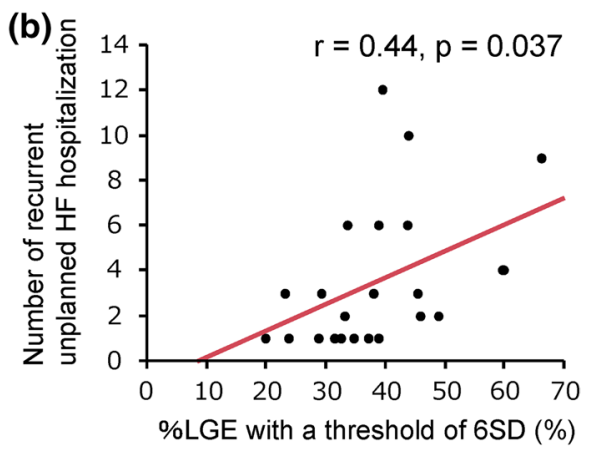

talizations. The number of recurrent hospitalizations per patient was related not to LVEF ( $r=-0.06, p=0.798$ ) but to \% LGE with a threshold of $6 \mathrm{SD}(r=0.44 ; p=0.037)$. Abbreviations as in Table 1 